\title{
Real-Time Parameter Identification of Hyperelastic Materials in a Closed Loop System Using Tactile Feedback
}

\author{
Ali Reza Hassan Beiglou, Siamak Arbatani, and Javad Dargahi
}

\begin{abstract}
Real time identification of materials with nonlinear characteristics is a challenging task. One of the characteristic which is very challenging to model is the stress relaxation behavior, which results in nonlinearity in mechanical behavior of material. A novel method is proposed in this research for real-time identification of materials possessing this behavior during Telemanipulation using Fuzzy logic algorithm. The proposed algorithm is evaluated in an experimental setup consisting a five degrees of freedom serial manipulator (Catalyst-5T) equipped with a strain-gauge sensor with a ball-caster tip. The system is able to detect surface of the material, then perform the identification task by implementing a specified depth of indentation on material and sliding horizontally on surface of the material while maintaining the applied indentation depth. Based on the real time data of stress relaxation time and measured force of indentation as inputs of Fuzzy material identification algorithm, material type is identified in real-time. Output of the system is crisp value which indicates the material type. Proposed algorithm is validated in a designed experimental scenario which consists of three different materials. Experimental results confirm reliability and precision of the proposed algorithm in material type discrimination.
\end{abstract}

Index Terms-Robotics, strain-gauge sensor, hyperelastic material, fuzzy algorithm, stress relaxation, real time.

\section{INTRODUCTION}

This subject of real time material identification for the materials with specific characteristics usually associates with tissue identification in minimal invasive robotic surgery. Lack of haptic feedback in robotic assisted surgery, usually leads the researchers to compensate this drawback by proposing different methods of material identification recreation of their mechanical behavior. Many researchers have used systems which are combination of a serial manipulator and a feedback sensor to simulate robotic surgery operations and also for different other purposes such as tracking a surface and obtaining behavior of soft tissues. Wang et al. equipped a 5 degrees of freedom serial manipulator with a tactile sensor and tried to use the tactile feedback sensor for tacking of a surface [1]. They use inverse kinematic model of robot to control position of robot's end-effector, and tried to keep the end-effector perpendicular to object surface using a hybrid impedance controller. Sangpradit et al. used the method of rolling over surface of soft tissue to locate hidden

Manuscript received November 15, 2013; revised March 12, 2014.

The authors are with the Department of Mechanical and Industrial Engineering, Concordia University, Montreal, Canada (e-mail: alir_has@encs.concordia.ca, dargahi@encs.concordia.ca). abnormalities under tissue. They tried to overcome the lack of haptic feedback in minimally invasive surgery by means of tissue discrimination. They employed Silicone Phantom in their experiment which possess Hyperelastic mechanical behavior, and modeled it in ABAQUS ${ }^{\mathrm{TM}}$ [2]. Hongbin et al. applied a method to identify the internal structure of soft tissue using a wheeled probe to generate a mechanical image by rolling across the surface of organs. First by dynamic analysis of wheel-tissue interaction, they obtained the parameters of lower layer tissue stiffness. Then, dynamic analysis is performed by capturing the information of material surface according to the developed mechanical image from rolling data, and characterizing the material according to force and tissue deflection [3]. Force modeling for needle insertion into soft tissue using a one degrees of freedom robot and a force sensor is performed by Allison et al. [4]. Stress relaxation is a mechanical property which is seen in soft tissues. In recent researches, this characteristic is not taken into account for acquiring the mechanical properties of materials. This research proposes a method for identification of the materials using force feedback and considering stress relaxation behavior which results in varying force feedback signal.

A five degrees of freedom robot (5 DOFs) is equipped by a strain-gauge sensor with ball caster tip, which is in contact with materials to be identified [5], [6]. Robot is controlled by inverse kinematic approach and a PD controller. In first step, force feedback of sensor is used to find the surface of material with PID control by robot. In this step, robot approaches to surface of the soft material up to a certain contact force $(0.01 N)$. In second step, robot implements an indentation to a given depth. In third step, robot will move horizontally on predefined on surface of the material, maintaining the depth of indentation which was applied in second step. During these two steps, both the vertical motion for indentation and also horizontal sliding on material, three parameters of indentation depth, applied force and duration of stress relaxation are fed to a smart Fuzzy material identification algorithm. This algorithm identifies the material type in real-time as robot slides on material surface, and shows the type of the materials which are previously assigned in training phase of the algorithm.

Rest of paper organized in four sections. Explanation of each part of system and designed fuzzy algorithm is discussed in Section II. In Section III the experiment setup which consisted of making a close loop with sensor and robot is presented. Result and discussion about input and output signals are presented in Section IV. Conclusion discussed in Section V. 


\section{Methodology}

According to basis of strength of material the traction vector is defined in (1). This equation states that the normal traction vector can be achieved considering action force over surface element.

$$
t^{n}=\lim _{s \rightarrow 0} \frac{\Delta F}{\Delta S}
$$

According to Cauchy's stress Lemma (2) the surface traction vector acts on opposite sides of surface and has the opposite direction relative to traction vector while the magnitude is the same. In fact, Cauchy's stress Lemma restates Newton's third law of motion for action and reaction forces.

$$
t^{n}=-t^{(-n)}
$$

Cauchy's law states that for each traction vector which acts on a surface, the Cauchy stress tensor can be defined by (3).

$$
t=\sigma n, t_{i}=\sigma_{i j} n_{j}
$$

Equation (4) shows the expansion of Cauchy's law formulation.

$$
\begin{aligned}
& t_{1}=\sigma_{11} n_{1}+\sigma_{12} n_{2}+\sigma_{12} n_{3} \\
& t_{2}=\sigma_{21} n_{1}+\sigma_{22} n_{2}+\sigma_{23} n_{3} \\
& t_{3}=\sigma_{31} n_{1}+\sigma_{32} n_{2}+\sigma_{33} n_{3}
\end{aligned}
$$

Considering Cauchy law, components of the stress tensor can be achieved in Cartesian space by (5) where the $i^{\text {th }}$ component of the traction vector acts on a surface which is normal to $e_{j}$.

$$
\delta_{i j}=e_{i} \sigma e_{j}=e_{i} \cdot t^{\left(e_{j}\right)}
$$

Stress tensor is illustrated in Fig. 1.

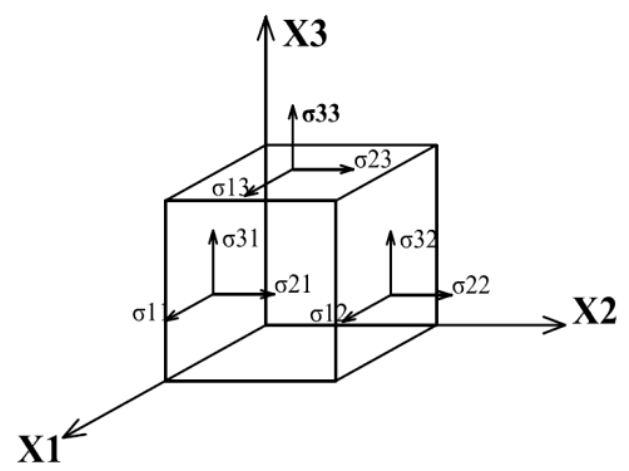

Fig. 1. Cauchy's stress tensor.

Young's modulus correlates the stress which is produced due to the traction vector on a surface with strain of material which is also the consequent of applying force on surface of material (6).

$$
\sigma=E \varepsilon
$$

Equation (6) shows that there is a linear relationship between the applied stress on surface of material and strain which is occurred because of change in length of material.
In this research we are interested to identify the type of material with some special characteristics using the phenomenon which is stated in (6). First assumption is that our materials are isotropic. Therefore, the linearity assumption which is presented in (6) can be taken into the account.

Traction vector and consequently the stresses are produced by implementing the normal indentation of specified area on surface of material. Materials which will be identified by smart algorithm are hyperelastic materials [7]. These materials have some special characteristics such as property of stress relaxation. Since stress relaxation leads to have varying signal for force, the intelligent material detection algorithm should be smart to recognize the stabilized stress signal. This reorganization is able by knowing the period of stabilization time for each material type. By knowing stabilization period, the smart algorithm will compare the period of stress relaxation which is recorded continuously through a designed switch by time periods which are used for training of detection algorithm. As the stabilized force signals are used as one of inputs of algorithm, first three experiments are designed to show that how linearity assumption can be used for these force values. Three types of hyperelastic materials are employed in this research for identification. These hyperelastic materials are ICF, E1 and EVA. In these experiments first, the system (robot which is equipped with a sensor) will find the surface of material. Touching force is defined equal to $0.01 \mathrm{~N}$. The procedure of finding the surface and implementing indentation on the surface of material is explained in part 3 by detail. After finding the surface, the system implements 3 different indentations on surface of material equal to $1 \mathrm{~mm}, 2 \mathrm{~mm}$ and $3 \mathrm{~mm}$. After implementing each indentation on surface of material, the designed switch records a period of time which is passed to get the stabilized force signal. Then, the stabilized stress values for each indentation are recorded. This experiment is done for each Hyperelastic material. Fig. 2 illustrates the stabilized force of sensor in terms of indentation which robot has applied on surface of material for ICF with indentation depths of 1,2 and $3 \mathrm{~mm}$. It can be clearly seen that assumption of having linear relationship between strain and stress which is previously discussed is acceptable. As a result, there is also a linear relation between the applied force and indentation depth. The same graphs are shown for material type 2, E1, in Fig. 3 and material type 3, EVA, in Fig. 4.

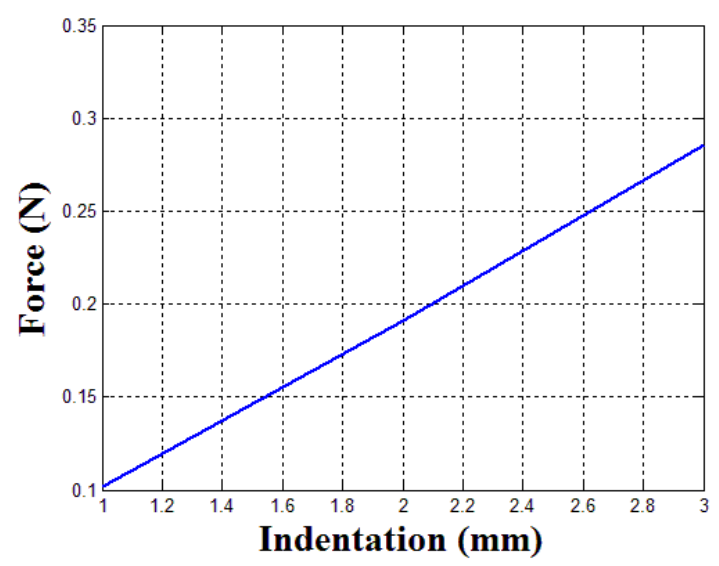

Fig. 2. Indentation-Force material type 1, ICF. 
The smart detection algorithm which is designed to identify the material type is an intelligent Fuzzy algorithm. Mamdani's method which is considered as one of direct methods of Fuzzy reasoning is used to identify the material type [8]. The indentation which is implemented on surface of material is applied by a five degrees of freedom robot arm, Catalyst-5T. Exact value of indentation is measured by robot's encoder in a joint space-Cartesian translation. The error which is defined as difference of the desired indentation and the indentation which is applied by robot is also considered as inputs of the Fuzzy detection algorithm.

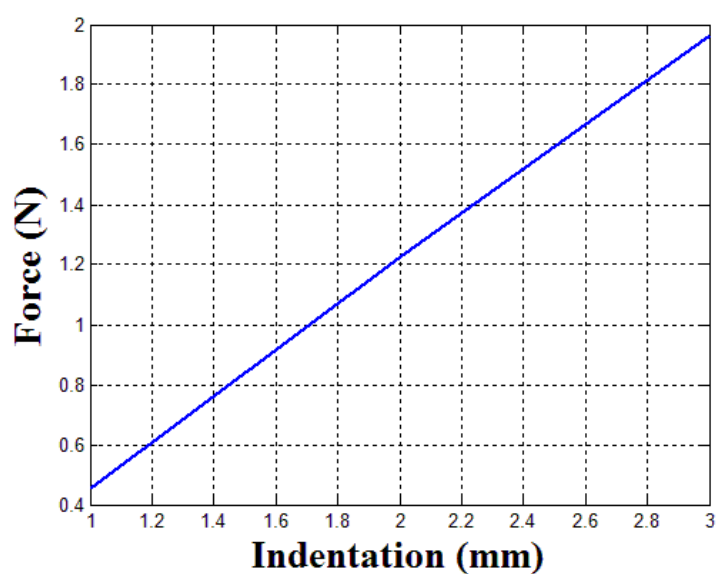

Fig. 3. Indentation-Force material type 2, E1.

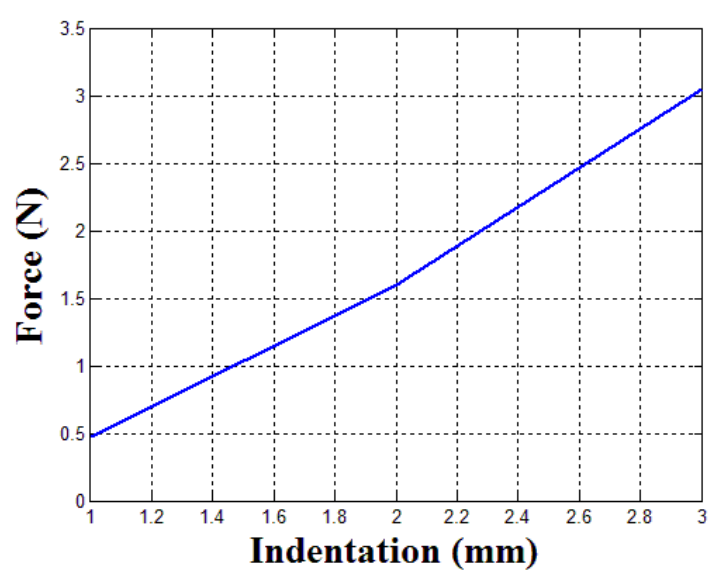

Fig. 4. Indentation-Force material type 3, EVA.

There are three parameters which are assigned for detection algorithm as the inputs of the system. Measured force value through strain gauge, implemented indentation by robot and time period for force stabilization measured by stabilization detection switch which is shown in Fig. 8. Implemented indentation, time period and applied force are shown in Fig. 5, Fig. 6 and Fig. 7.

As shown in Fig. 5, 6 and 7, the inputs of the system are defined as Fuzzy triangular sets. Based on the distribution of gathered data the margin of Fuzzy stets are defined for each input of the system. According to material thickness which is used in presented case study, a maximum of $5 \mathrm{~mm}$ of indentation can be applied, in which each millimeter is defined as a Fuzzy input shown in Fig. 5. The stress relaxation is the main reason of defining the time dependant system Fig. 6 shows the time distribution of stress relaxation for materials. The main input of the detection algorithm is range of measured force related to each material which is defined as third input of the system in Fig. 7.

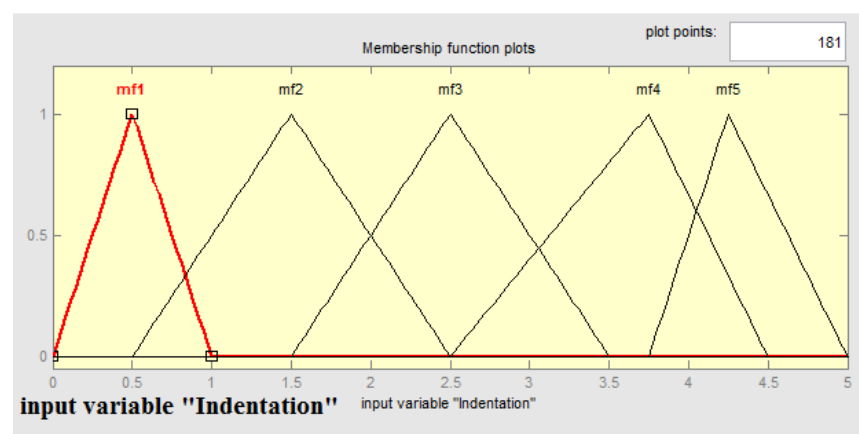

Fig. 5. Indentation input of fuzzy algorithm.

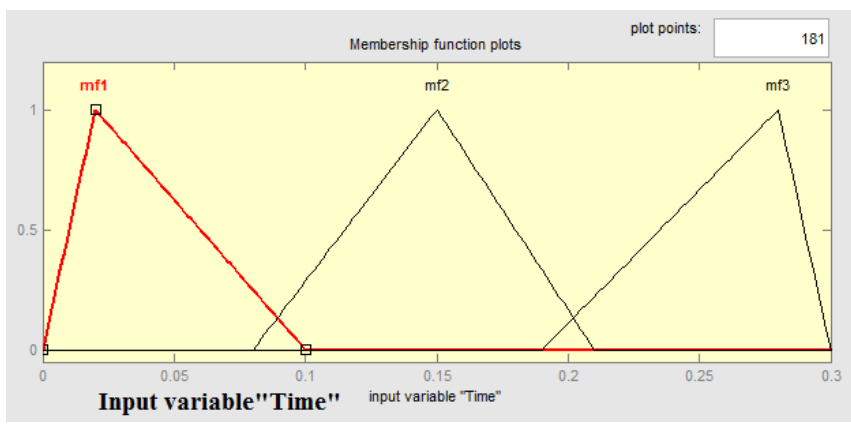

Fig. 6. Time period input of fuzzy algorithm.

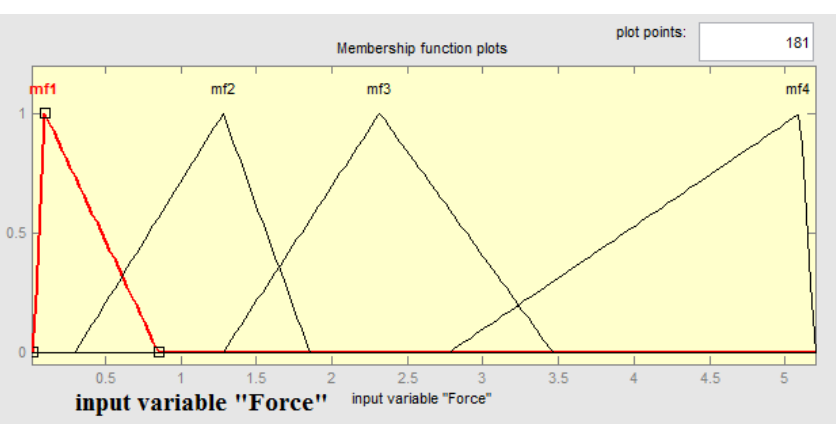

Fig. 7. Force input of fuzzy algorithm.

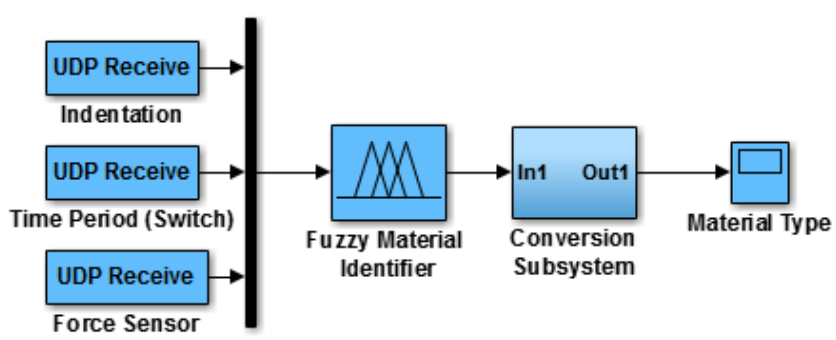

Fig. 8. Fuzzy algorithm inputs/output.

Since there are three types of materials which will be identified, the Fuzzy detection output is defined as a range of numbers which are varied through value of 1 to 3 . Therefore output of the Fuzzy detection algorithm has discrete values of 1, 2 and 3 after truncation. Each number refers to a material which previously assigned to it (Mat No.1: ICF, Mat No.2: E1, and Mat No.3: EVA). Fuzzy output is illustrated in Fig. 9.

Eighteen rules are defined to correlate a logical relationship between the inputs and output of Fuzzy material detection algorithm. The rules are presented as surface between each set of two input and output of the system which are shown in Fig. 9-Fig. 12. 


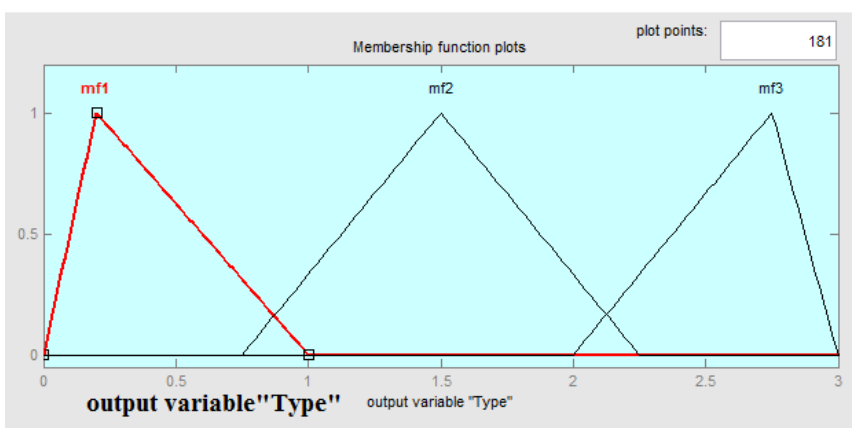

Fig. 9. Fuzzy algorithm output.

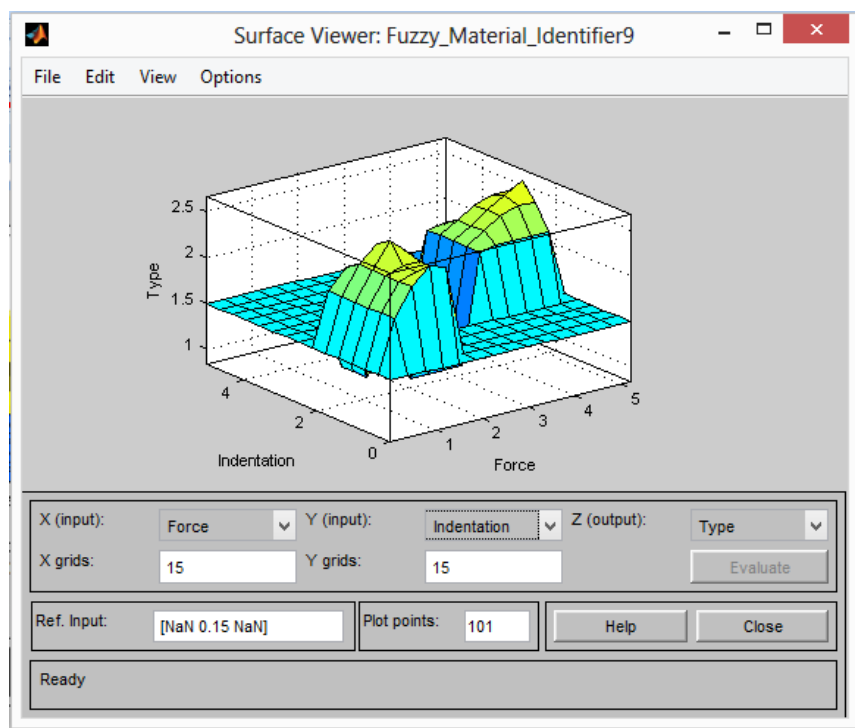

Fig. 10. Force-Indentation surface in fuzzy space.

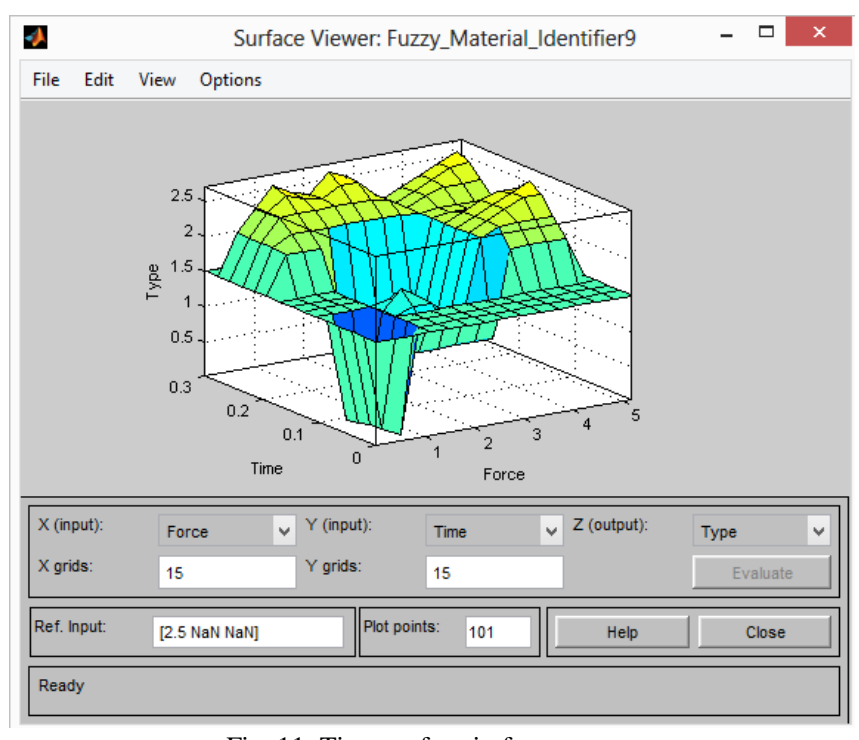

Fig. 11. Time surface in fuzzy space.

In order to defuzzify the output of the system Supermom of Maximum (SOM) method is used. In other words, maximum possible value in the universe discourse of the Fuzzy output which is related to point to the maximum Fuzzy membership is the output of the system in crisp logic.

Desired motion is in directed in material identification path. Desired direction of motion is shown in Fig. 15. During this motion, abovementioned inputs are fed to smart Fuzzy algorithm, using the defined rules. The algorithm outputs the number which represents the material which previously assigned to it.

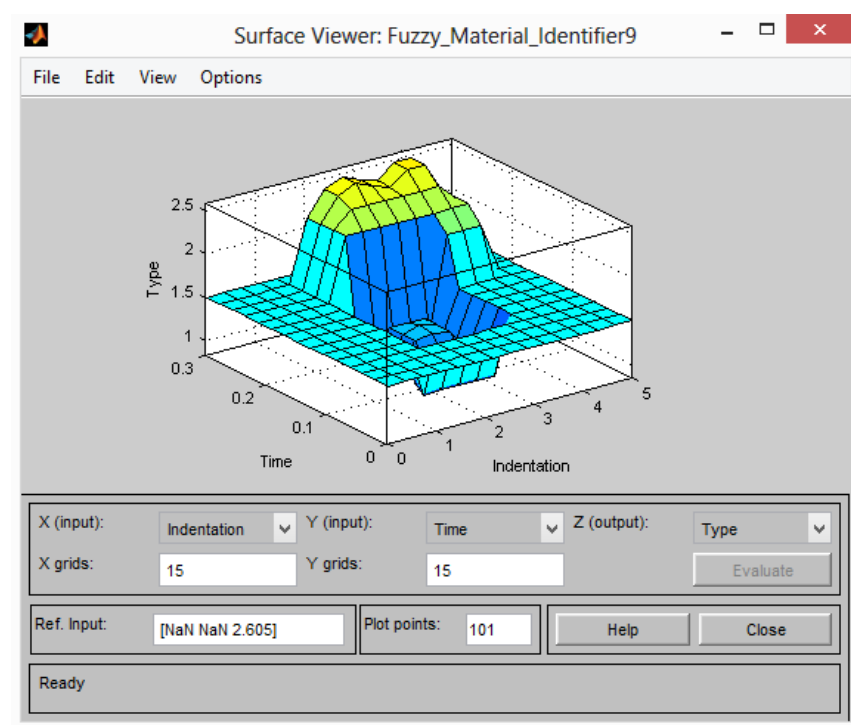

Fig. 12. Indentation-time surface in fuzzy space.

\section{EXPERIMENTAL SETUP}

In current research, a five degrees of freedom robot arm, Catalyst-5T, is employed which is equipped with a force sensor. The robot controlled by an inverse kinematic approach and a PD controller which is designed to enable robot to move in desired directions in a Cartesian space. A PD controller is used for each joint of the robot to achieve the proper angles of robot's arm in order to reach a desired gripper position in Cartesian space. Since controlling the robot itself needs high volume of mathematical operation in Matlab Simulink environment, two separate Matlab Simulinks are designed to distribute the volume of calculation between two computers over a Local Area Network (LAN). The stream of force data is transferred through an internal network using UDP (User Datagram Protocol) object in Matlab Simulink to input the Fuzzy detection algorithm. UDP is employed in order to minimize data transfer delays in network. The exact value of implemented indentation is measured by robot's encoder feedback. By implementing each indentation depth, the PD controller of robot tries to reach the reference point. Considering the steady state error of the controller, the exact implemented indentation is measured and fed to the detection algorithm. The switch or time period recorder provides the amount of time to the algorithm, which is taken to stabilize the force. This value is achieved through a clock object on Matlab Simulink. The clock will starts as the robot implements an indentation on surface of the material and consequently the sensor shows a sudden change in force value. As the material relaxation occurs, derivate of force regarding to time approaches to zero value. This derivative is used as an indicator for recognizing the stabilized stress time. A switch is employed for turning off the clock upon detecting the zero value from the force signal derivate. The recorded duration by clock indicates stress relaxation period. Fig. 8 shows the three Fuzzy inputs as well as the output of the Fuzzy detection algorithm.

Goal of this research is to identify the type of material when an indentation is occurred on surface of material, and also when the sensor is moving on surface of material by 
maintaining the implemented indentation depth. To measure the forces which are in range of $0.1 \mathrm{~N}$ to $4 \mathrm{~N}$ a strain-gauge is used [9]. Small changes of force, which is applied to the strain-gauge, result in change in amount of bending in strain-gauge. The resulted signal is acquired and transferred to Matlab Simulink environment by adata acquisition card (DAQ). In order freely slide on surface of any type of soft material, a ball caster is connected to one end of the strain-gauge (see Fig. 13).

Using ball caster leads to minimize the outliers in stream of force data, eliminating the shocks which are seen in first as horizontal motion starts, and also when the motion ends. Rolling on surface of material instead of frictional sliding, avoids sudden change in amount of force at the boundary of different materials. Fig. 14 shows the robot which is equipped with the strain-gauge sensor shown in Fig. 13.

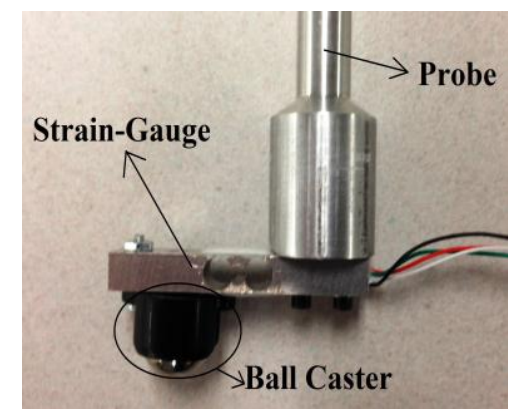

Fig. 13. Strain gauge-sensor.

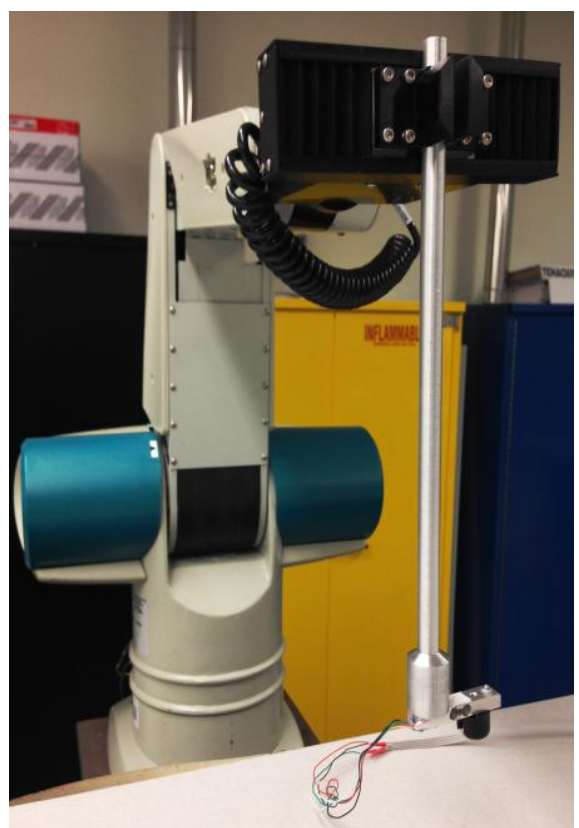

Fig. 14. Robot equipped with strain-gauge sensor.

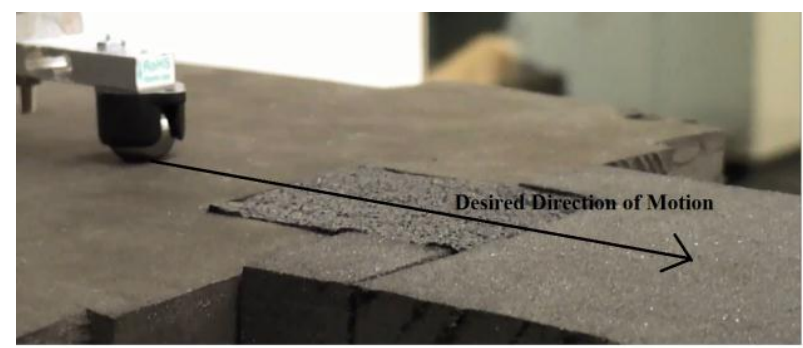

Fig. 15. Desired direction of motion, material (left to right): Type3: EVA, Type1: ICF, Type2: E1.

\section{RESUlTS AND DisCUSSION}

Fig. 16 shows the real-time input signal which is transferred to the Fuzzy algorithm. This signal is produced by carrying out a maneuver which is defined as implementing an indentation depth of $3 \mathrm{~mm}$ on surface of material and moving on a desired direction by maintaining the indentation depth. During this maneuver the PD controller of robot tries to maintain the desired indentation depth on surface of material despite the fact that during this maneuver as the material type changes, the toughness of material varies.

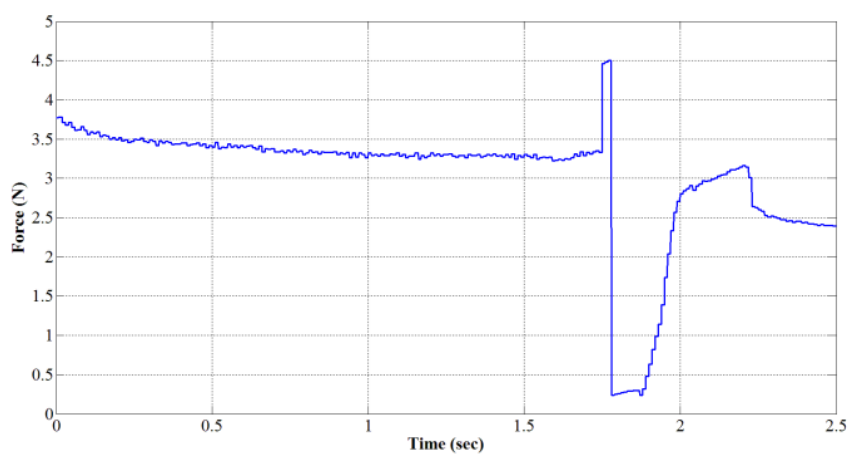

Fig. 16. Input signal.

As mentioned, three parameters are inputs of the proposed algorithm. These parameters are force, indentation depth and relaxation time period. Force variation with time is presented in Fig. 16. Indentation depth is acquired from robot arm encoders and the relaxation time is measured by the switch mentioned above. As the three inputs enter the detection algorithm, all Fuzzy rules which are discussed in methodology are checked and number of material which was assigned to it will be shown as output.

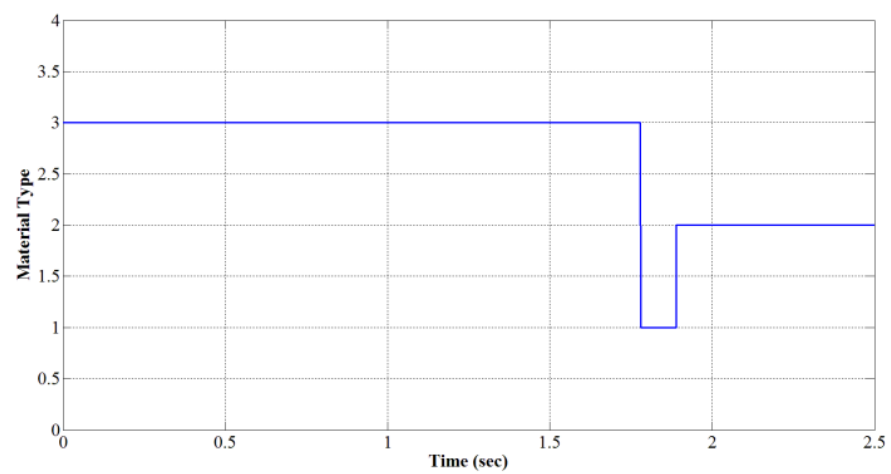

Fig. 17. Fuzzy detection algorithm output.

The maneuver which is illustrated in Fig. 15, results in signals shown in Fig. 16 and Fig. 17 which illustrate the input signal of force and material type output in time space, respectively. It can be seen that while the force which is transferred to the detection algorithm lays in the range of the material number three (EVA), and the recorded stress relaxation period becomes equal to the value which is achieved during the previously training phase , the output will be "number three" which is assigned to material EVA. This motion takes about $1.6 \mathrm{sec}$. As a result, the detection algorithm's output will show the material type three from beginning of maneuver for $1.6(\mathrm{sec})$. After this period, the switch will update the period of time for new material which is indented by ball caster. The same reasoning is investigated for two other materials, ICF and E1 which are shown in Fig. 15 
for applied maneuver.

\section{CONCLUSION}

This paper presents a method for identification of materials with mechanical stress relaxation behavior using a force feedback strategy considering the time period of stress relaxation with a smart Fuzzy detection algorithm. Three different Hyperelastic materials are selected for identification by a system which is consisted of a robot that equipped with a strain-gauge sensor and the proposed algorithm. First, some experiments are designed and done to show that traction vector of surface and indentation has linear behavior in range of implemented indentation. An experiment is performed to show that the proposed intelligent Fuzzy algorithm is capable of identifying the material type with stress relaxation characteristics. The results demonstrated that designed Fuzzy detection method can identify the material type accurately with an acceptable delay in a real time manner.

\section{REFERENCES}

[1] J. Wang and Y. Li, "Surface-tracking of a 5-DOF manipulator equipped with tactile sensors," in Proc. 11th Int, Conf. Control, Automation, Robotics and Vision (ICARCV), Singapore, 2010, pp. 2448-2453.

[2] K. Sangpradit, H. Liu, L. D. Seneviratne, and K. Althoefer, "Tissue identification using inverse finite element analysis of rolling indentation," in Proc. IEEE International Conference on Robotics and Automation (ICRA), Kobe, 2009, pp. 1250-1255.

[3] H. Liu, D. P. Noonan, K. Althoefer, and L. D. Seneviratne, "Rolling mechanical imaging: A novel approach for soft tissue modelling and identification during minimally invasive surgery," in Proc. IEEE International Conference on Robotics and Automation (ICRA), Pasadena, 2008, pp. 845-850.

[4] A. M. Okamura, C. Simone, and M. D. O'Leary. (October 2004). Force modeling for needle insertion into soft tissue. IEEE Trans. Biomedical Engineering. [Online]. 51(10). pp. 1707-1716. Available: http://ieeexplore.ieee.org/xpls/abs_all.jsp?arnumber=1337139

[5] J. A. Velarde-Sanchez, S. A. Rodriguez-Gutierrez, L. G. Garcia-Valdovinos, and L. G. Pedraza-Ortega, "5-DOF manipulator simulation based on MATLAB-Simulink methodology," in Proc. 20th International Conference on Electronics, Communications and Computer (CONIELECOMP), Cholula, 2010, pp. 295-300.

[6] A. Z. Alassar, I. M. Abuhadrous, and H. A. Elaydi, "Modeling and control of 5 DOF robot arm using supervisory control," in Proc. 2nd IEEE International Conference on Advanced Computer Control (ICACC), Singapore, 2010, pp. 351-355.

[7] S. Hartmann and P. Neff. (June 2003). Polyconvexity of generalized polynomial-type hyperelastic strain energy functions for near-incompressibility. International Journal of Solids and Structures. [Online]. 40(11). pp. 2767-2791. Available: http://www.sciencedirect.com/science/article/pii/S002076830300086 6
[8] H. Zhou and H. Ying. (June 2013). A method for deriving the analytical structure of a broad class of typical interval type-2 mamdani fuzzy controllers. IEEE Transactions on Fuzzy Systems, [Online]. 21(3). pp. 447-458. Available: http://ieeexplore.ieee.org/xpl/articleDetails.jsp?arnumber=6341818

[9] D. M. Stefanescu, A.T. Farcasiu, and A. Toader. (October 2012). Strain gauge force transducer and virtual instrumentation used in a measurement system for retention forces of palatal plates or removable dentures. IEEE Sensors Journal. [Online]. 12(10). pp. 2968-2973. Available:

http://ieeexplore.ieee.org/xpl/articleDetails.jsp?arnumber=6227327

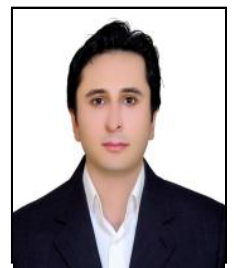

A. R. Hassan Beiglou has graduated in BSc of mechanical engineering in Iran. He has 5 years work experience in designing and stress analysis of static equipments in oil, gas and petrochemical industries. Mr. Hassan Beiglou joined as a MSc research associate with Dr. Dargahi's research team in September 2013. His research interest is in area of mechatronics, sensors, and haptic feedback in simulating robotic surgery.

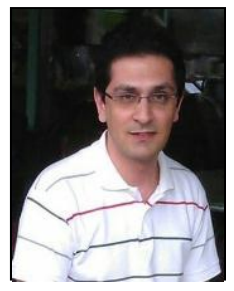

S. Arbatani received a scholarship to conduct his MSc degree in Mechanical Engineering Department at Shiraz University. He completed his degree with the rank of second best student in the entire department. He worked for the Concept Software Company for couples of years. Mr. Arbatani joined as a PhD research associate with Dr. Dargahi's research team in January 2011. His research interest is in area of haptic feedback in robotic assisted minimal surgery, specifically in development of state of art haptic displays.

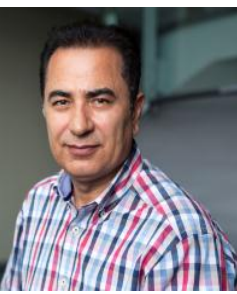

J. Dargahi serves as a full-professor in the Mechanical and Industrial Engineering Department at Concordia University in Montreal, Canada. $\mathrm{He}$ received his B.Sc. and M.Sc. degree in mechanical engineering from University of Paisley, UK and his Ph.D. degree from Glasgow Caledonian University, UK in the area of "Robotic Tactile Sensing". Prof. Dargahi has published over 150 refereed journal and conference papers. He is the author of three new books published by McGraw-Hill and Wiley. He has also a patent in the area of tactile sensing and displays. Dr. Dargahi has been principal reviewer of several major NASA proposals in the area of "Crew health and performance in space exploration mission". 